\title{
Use of fluoxetine in patients with diabetes mellitus
}

\author{
Gill Salmon
}

\begin{abstract}
Although pharmaceutical companies report side effects that may occur with use of their medicotions, Ilitie data on speclitc groups of patients that may be at itsk of particular side efiects is ovallable (Katz of al, 1991). Patients with diabotes mellitus form an important group for pesychiatilsts since the occurrence of depression is relatively common with this group and guidance on its treatiment is lacking.
\end{abstract}

The manufacturers of fluoxetine suggest that the drug is used with caution in patients with diabetes mellitus and state in the data sheet that in these patients, fluoxetine may alter glycaemic control. They advise that insulin and/or oral hypoglycaemic dosage may thus need to be altered.

Most of the problems reported with fluoxetine relate to non-insulin dependent diabetes mellitus (NIDDM, type 2 disease, adult-onset) and not the less common insulin dependent form (IDDM, type 1 disease, juvenile-onset). Studies have shown that fluoxetine improves peripheral and hepatic insulin action in NIDDM irrespective of its weight lowering effect (Kutnowskd et al, 1992; Potter van Loon et al, 1992). It is conceivable too, that abnormal pancreatic function in the pre-diabetic or the unrecognised diabetic patient receiving fluoxetine could result in a lowering of blood glucose (hypoglycaemia). Lear \& Burden (1992) reported a case in which an insulin dependent diabetic patient started on fluoxetine was convinced she was having repeated hypoglycaemic attacks because of the onset of symptoms of nausea, tremor, sweating, anxiety and lightheadedness. This was despite a laboratory blood glucose of $13 \mathrm{mmol} / \mathrm{l}$, unchanged insulin requirements and the episodes disappearing when the drug was stopped. In diabetic patients who complain of symptoms of hypoglycaemia when started on fluoxetine, it is thus important to confirm the diagnosis before altering medication.
Katz et al (1991) described the clinical course of three patients with NIDDM who experienced alterations in glucose control after exposure to fluoxetine. Acute and chronic use of fluoxetine was linked with improved glucose control and/or hypoglycaemia while discontinuation could result in hyperglycaemia. It was thought that possible explanations for these disturbances were likely to be found in studies relating to serotonin $(5-\mathrm{HT})$ and the hypothalamic-pituitary-adrenal axis, 5-HT and catecholamine neuronal activity, and finally neurotransmitter concentrations and pancreatic secretion of glucagon and insulin. One of the mechanisms proposed highlighted the role of $5-\mathrm{HT}$ in the secretion of glucocorticoid, reduction of neuronal transmission of the former (as occurs with regular use of fluoxetine) being associated with a reduction in the latter in rats (Ahren et al, 1986). Glucocorticoid secretion participates in the regulation of serum glucose and an elevated level of glucocorticoid might explain the occurrence of hyperglycaemia on withdrawal of fluoxetine. Thus the use of fluoxetine in NIDDM may destabilise the control of serum glucose concentrations by changing central and peripheral activities of 5-HT, noradrenaline, adrenaline and acetylcholine, and certain populations of patients may be at increased risk from what would otherwise be a rare side effect.

When treating diabetic patients with fluoxetine, the clinician should consider cautioning them about these side-effects and regularly checking serum glucose levels. The benefits of using this drug over other antidepressant medications, however, include increased compliance because of its anorectic and weight reducing properties and a lower incidence of anticholinergic side effects. 


\section{References}

AHREN, B.. TABORSKY, Jr, G. J. \& PORTE, Jr, D. (1986) Neuropeptide versus cholinergic and adrenergic regulation of islet hormone secretion. Diabetologia, 29. 827-836.

LEAR. J. \& BURDEN, A. C. (1992) Fluoxetine side-effects mimicking hypoglycaemia (letter). Lancet, 3s9, 1296.

KaTZ. L. M.. Fochmmann. L. J. \& PATO. M. T. (1991) Clomipramine, fluoxetine and glucose control. Annals of Clinical Psychiatry, 3/4, 271-274.

Kutnowsk, M., DAubresse, J. C.. Friedman, H., et al (1992) Fluoxetine therapy in obese diabetic and glucose intolerant patients. International Journal of Obesity. 16(Suppl. 4), 63-66.

POTTER VAN LOON, B. J., RADDER, J. K., Frouch, M., et al (1992) Fluoxetine increases insulin action in obese type 11 (non-insulin dependent) diabetic patients. International Journal of Obesity. 16(Suppl. 4), 55-61.

Gill Salmon, Senior Registrar in Child and Adolescent Psychiatry, Child and Family Service, Brookside Clinic, Station Way. Aylesbury, Bucks HP2O 2SQ 